\title{
POTENCIALIDADES DO USO DE ULTRASSOM NA DETERMINAÇÃO DO PESTICIDA CARBARIL EMPREGANDO ELETRODOS DE DIAMANTE ${ }^{\#}$
}

\author{
Gustavo S. Garbellini* e Luis A. Avaca \\ Instituto de Química de São Carlos, Universidade de São Paulo, CP 780, 13560-970 São Carlos - SP, Brasil \\ Giancarlo R. Salazar-Banda \\ Instituto de Tecnologia e Pesquisa, Universidade Tiradentes, 49032-490 Aracaju - SE, Brasil
}

Recebido em 16/12/09; aceito em 8/6/10; publicado na web em 18/10/10

\begin{abstract}
ULTRASOUND POTENTIALITIES ON THE DETERMINATION OF THE PESTICIDE CARBARYL USING DIAMOND
ELECTRODES. The potentiality of the use of ultrasound radiation in association with a boron-doped diamond electrode was evaluated on the voltammetric determination of the pesticide carbaryl. Improvements in the sensitivity, limit of detection and reproducibility of the measurements were observed due to both, the enhancement of mass transport and the cleaning of the electrode surface provided by ultrasound. Satisfactory recovery levels for carbaryl in pure water (96-98\%) and pineapple juice (89-92\%) for quiescent and sonovoltammetric methodologies were obtained. These methodologies can be alternative tools for the analyses of pesticides in fruit samples, mainly the insonated condition that improve the analytical performance and dispense intermediary cleanings of the electrode surface.
\end{abstract}

Keywords: boron-doped diamond; sonoelectroanalysis; food samples.

\section{INTRODUÇÃO}

A determinação eletroanalítica direta de compostos orgânicos tóxicos, como os pesticidas, em amostras de alimentos é fortemente prejudicada pela adsorção e/ou insolubilização de produtos da oxidação destes compostos na superfície de eletrodos e, também, pela grande quantidade de interferentes presentes nessas amostras, diminuindo deste modo a sensibilidade e a reprodutibilidade das medidas eletroquímicas.

Uma possibilidade de minimizar esse fenômeno de inativação eletródica é o uso da radiação de ultrassom em associação a estudos voltamétricos. ${ }^{1,2} \mathrm{~A}$ introdução do ultrassom em sistemas eletroquímicos é caracterizada pelo significativo aumento do transporte de massa que melhora a sensibilidade analítica em comparação a medidas quiescentes. O uso de ultrassom promove também a ativação da superfície eletródica por meio do processo de cavitação, que consiste na criação, ampliação e implosão de cavidades de vapor e gases. ${ }^{3}$ Esta melhora do transporte de massa é devida ao colapso de bolhas perto ou na interface eletrodo-solução, com a formação de fluxos de altas velocidades direcionados à superfície eletródica e à movimentação da bolha na região próxima ou dentro da camada de difusão do eletrodo. É interessante ressaltar que os fluxos acústicos são os maiores responsáveis pelo fluxo convectivo até a interface sólido-líquido. Diante disso, o aumento do transporte do material eletroativo à superfície eletródica pode ser medido assumindo uma diminuição da espessura da camada de difusão $(\delta){ }^{4}$

Esses efeitos sinérgicos do ultrassom foram demonstrados na determinação de nitrito em água pura ${ }^{5}$ e de pesticidas e subprodutos (metilparation e 4-nitrofenol) em amostras de alimentos ${ }^{6,7}$ sobre o eletrodo de diamante dopado com boro (DDB). Desta maneira, decidiu-se explorar a potencialidade da associação do ultrassom ao eletrodo de DDB para a determinação do pesticida carbaril (pertencente ao grupo dos carbamatos). Codognoto et al. ${ }^{8}$ mostraram a determinação de carbaril em águas naturais via oxidação do nitrogênio amida da molécula do composto, utilizando a voltametria de onda quadrada (SWV) e o eletrodo de DDB.

*e-mail: gustgarb@yahoo.com.br

"Artigo em homenagem ao Prof. Hans Viertler
Esse processo se mostrou irreversível (ausência de corrente reversa) e ocorreu num potencial de $1,45 \mathrm{~V}$ vs. $\mathrm{Ag} / \mathrm{AgCl}$. Essa determinação voltamétrica foi prejudicada pela forte adsorção e/ou insolubilização de produtos da oxidação de carbaril na superfície do eletrodo.

O carbaril (1-naftil $N$-metilcarbamato), inseticida de amplo espectro, é usado para o controle de pragas em várias culturas como abacaxi, algodão, maçã, tomate, entre outras, além do uso como moluscicida e acaricida. ${ }^{9}$ A exposição dos humanos a este composto pode causar a inibição da enzima colinesterase, originando problemas neurológicos. ${ }^{10}$ Geralmente, a pele humana apresenta uma larga absorção desse pesticida. ${ }^{11}$ Diante da necessidade do monitoramento de resíduos deste composto, a Agência Nacional de Vigilância Sanitária (ANVISA) $^{12}$ estabeleceu os limites máximos de resíduos (LMRs) permitidos para o carbaril em diferentes culturas agrícolas, sendo o valor, por exemplo, para a cultura de abacaxi de $500 \mu \mathrm{g} \mathrm{kg}^{-1}$.

Os resíduos de carbaril em amostras ambientais são geralmente determinados por técnicas cromatográficas ${ }^{13-17}$ que incluem complicados pré-tratamentos ou clean-up das matrizes. Uma alternativa para essas análises são as técnicas eletroanalíticas empregando, por exemplo, eletrodos modificados ${ }^{18-22}$ e DDB..$^{8,23}$ Esse material eletródico ${ }^{24-28}$ apresenta ampla janela eletroquímica devida à pouca reatividade química da superfície do diamante, grande resistência a vários meios agressivos, baixas correntes capacitivas e robustez mecânica, que o caracteriza como um material ideal para experimentos sonoeletroquímicos. ${ }^{29,30}$

Com isso, o objetivo deste estudo foi avaliar os comprovados efeitos benéficos do ultrassom em eletroquímica, na determinação voltamétrica de carbaril em água pura e em uma matriz complexa (amostra de suco de abacaxi) empregando também o eletrodo de diamante.

\section{PARTE EXPERIMENTAL}

\section{Reagentes e soluções}

Foi preparada uma solução estoque do carbaril (Bayer Crop Science, $99,3 \%)$ na concentração de $1,0 \times 10^{-2} \mathrm{~mol} \mathrm{~L}^{-1}$ em acetonitrila (J.T. Baker). O eletrólito suporte utilizado foi o $\mathrm{Na}_{2} \mathrm{SO}_{4}$ (J.T. Baker) $0,1 \mathrm{~mol} \mathrm{~L}^{-1}$. 


\section{Preparo da amostra}

A amostra de abacaxi foi adquirida no comércio local. A determinação de resíduos de carbaril no suco desta amostra foi realizada após um procedimento de extração líquido-líquido (ELL). ${ }^{31}$ Este consistiu do uso de $10 \mathrm{~g}$ da amostra bruta de abacaxi e de $10 \mathrm{~mL}$ de acetonitrila. A amostra foi fortificada com uma quantidade de carbaril, resultando em uma concentração de $5,0 \times 10^{-3} \mathrm{~mol} \mathrm{~L}^{-1}$ do composto. A mistura (amostra fortificada + acetonitrila) foi agitada por $2 \mathrm{~min}$ e deixada em repouso por $30 \mathrm{~min}$.

\section{Procedimento analítico}

Previamente à construção de curvas analíticas na ausência e na presença do ultrassom, o eletrodo de DDB recebeu um pré-tratamento anódico a $+3,0 \mathrm{~V}$ e catódico a $-3,0 \mathrm{~V}$ durante 5 e $30 \mathrm{~s}$, respectivamente, em uma solução de $\mathrm{H}_{2} \mathrm{SO}_{4} 0,5 \mathrm{~mol} \mathrm{~L}{ }^{-1}$, para garantir a reprodutibilidade das medidas eletroquímicas ${ }^{25}$ e a obtenção de baixos limites de detecção (LD). ${ }^{32}$ As curvas analíticas foram obtidas pela adição de quantidades conhecidas do analito padrão à solução contendo o eletrólito suporte.

Para a determinação do limite de detecção e de quantificação (LQ), ${ }^{33}$ utilizou-se o desvio-padrão da média aritmética de oito voltamogramas dos brancos obtido das correntes medidas $\left(\mathrm{S}_{\mathrm{B}}\right)$ no mesmo potencial do pico voltamétrico do carbaril e as Equações 1 e 2 , onde $b$ corresponde ao coeficiente angular das curvas analíticas.

$$
\begin{aligned}
& \mathrm{LD}=3 \mathrm{~S}_{\mathrm{B}} / \mathrm{b} \\
& \mathrm{LQ}=10 \mathrm{~S}_{\mathrm{B}} / \mathrm{b}
\end{aligned}
$$

Os experimentos de recuperação foram realizados adicionando-se também alíquotas fixas do analito às soluções contendo somente o eletrólito suporte e às soluções contendo o eletrólito e uma quantidade fixa da amostra de suco após a ELL, seguida por adições de quantidades conhecidas do analito padrão e com isso, obtiveram-se as curvas analíticas resultantes. As concentrações analisadas de carbaril foram de 9,0 e 30,0 × $10^{-6} \mathrm{~mol} \mathrm{~L}^{-1}$. As porcentagens de recuperação ( $\mathrm{R} \%$ ) para o processo na ausência e na presença do ultrassom foram calculadas pela Equação 3, cujo o valor da concentração do analito encontrada se refere ao valor obtido pela extrapolação das curvas analíticas.

$$
\mathrm{R} \%=[\text { analito }]_{\text {encontrado }} /[\text { analito }]_{\text {adicionado }} \mathrm{x} 100
$$

\section{Instrumentação}

Foi utilizada uma célula eletroquímica de vidro Pyrex ${ }^{\circledR}$ de compartimento simples $(50 \mathrm{~mL})$ com entrada para a ponteira do ultrassom, para o tubo de borbulhamento de nitrogênio e para o sistema de três eletrodos. ${ }^{1}$ Foram empregados o eletrodo de DDB com 8000 ppm de $\mathrm{B}$ e de área geométrica de $0,25 \mathrm{~cm}^{2}$ como eletrodo de trabalho (Adamant Technologies SA, La Chaux-de-Fonds, Suíça), Ag/AgCl ( $\mathrm{KCl}$ 3,0 $\mathrm{mol} \mathrm{L}^{-1}$ ) como eletrodo de referência e uma placa de platina de $1,0 \mathrm{~cm}^{2}$ como eletrodo auxiliar. A ponteira do ultrassom foi colocada em frente ao eletrodo de DDB.

As medidas eletroquímicas foram realizadas utilizando-se um potenciostato da marca Autolab, modelo PGSTAT 30. A necessidade do controle bipotenciostático ${ }^{34}$ da ponteira de titânio do ultrassom foi eliminada, isolando o transdutor em relação à ponteira com um disco de Teflon ${ }^{\circledR}$. O ultrassom foi gerado por um equipamento modelo VCX 130 (Sonics \& Materials, EUA), capaz de emitir radiação em uma frequência fixa de $20 \mathrm{kHz}$.
A intensidade do ultrassom foi controlada pela amplitude de vibração da ponteira quando imersa em solução (20-50\%). A intensidade de $20 \%$ (potência máxima de $14 \mathrm{~W}$ ) foi aplicada em todo o estudo somente durante a realização de cada medida voltamétrica, para minimizar o aquecimento local da solução e prolongar a vida útil do eletrodo.

\section{RESULTADOS E DISCUSSÃO}

\section{Determinação sonovoltamétrica de carbaril em água pura}

Conforme relatado anteriormente ${ }^{8}$ a determinação de carbaril por SWV sobre o eletrodo de DDB é bastante prejudicada pela significativa adsorção e/ou insolubilização de produtos da oxidação do carbaril, contribuindo para a inativação da superfície do eletrodo.

Similarmente à detecção sonoeletroquímica de metilparation e de 4-nitrofenol, ${ }^{6,7}$ o ultrassom foi aplicado na determinação voltamétrica de carbaril em condições previamente otimizadas de meio reacional e de parâmetros da $S W V,{ }^{8}$ tais como frequência (f), amplitude (a) e incremento de varredura $\left(\Delta \mathrm{E}_{\mathrm{s}}\right)$, para minimizar a inativação eletródica e melhorar a sensibilidade da resposta voltamétrica. Estas condições foram $\mathrm{Na}_{2} \mathrm{SO}_{4} 0,1 \mathrm{~mol} \mathrm{~L}^{-1}(\mathrm{pH}=6,0)$ como eletrólito suporte com $\mathrm{f}$ $=300 \mathrm{~s}^{-1}, \mathrm{a}=50 \mathrm{mV}$ e $\Delta \mathrm{E}_{\mathrm{s}}=2 \mathrm{mV}$.

A metodologia sonoeletroanalítica consistiu na otimização de parâmetros, tais como a distância da ponteira do ultrassom em relação ao eletrodo $(d)$, a amplitude de vibração da ponteira quando imersa em solução $(A)$ e o tempo de aplicação da radiação antes das medidas eletroquímicas $(t)$. Vários voltamogramas em diferentes valores de $d$ (25-5 mm), $A$ (20-50\%) e $t(0,5$ a $20 \mathrm{~s})$ foram obtidos, comparados entre si e com a resposta obtida na ausência do ultrassom. Na Figura 1, estão apresentados os voltamogramas para a oxidação do carbaril na ausência e na presença do ultrassom para vários valores de $d$.

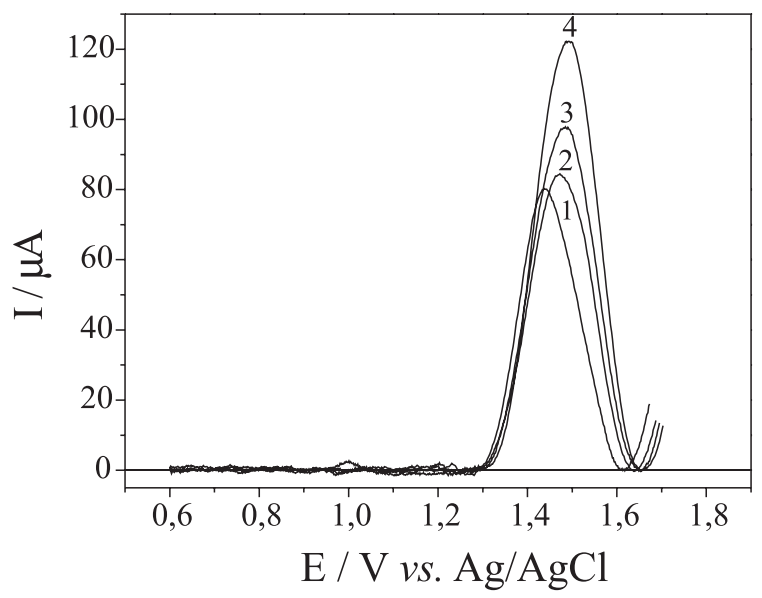

Figura 1. Sono-SWV mostrando o efeito da variação da distância da ponteira do ultrassom em relação ao eletrodo de DDB para a oxidação de carbaril a $50,0 \times 10^{-6}$ mol $L^{-1}$ na ausência (1) e na presença do ultrassom (2-4) sendo a d de 15 (2); 10 (3); $5 \mathrm{~mm}$ (4). Condições: $\mathrm{Na}_{2} \mathrm{SO}_{4} 0,1 \mathrm{~mol} \mathrm{~L} \mathrm{~L}^{-1}(\mathrm{pH}=6,0) \mathrm{com}$ $f=300 \mathrm{~s}^{-1}, a=50 \mathrm{mV}, \Delta E_{\mathrm{s}}=2 \mathrm{mV}$. Ultrassom: $A=20 \%$ e $t=0 \mathrm{~s}$

Notou-se que o valor da corrente dos sonovoltamogramas aumenta à medida que a $d$ diminui justificado pelo aumento do transporte do material eletroativo até a superfície eletródica e diminuição da $\delta$. Pela obtenção da maior intensidade da resposta sonovoltamétrica a uma $d$ de $5 \mathrm{~mm}$, este valor foi utilizado nos estudos posteriores. $\mathrm{O}$ estudo sonovoltamétrico não foi realizado em menores valores de $d$, devido à possibilidade de erosão da superfície do eletrodo de DDB e também ao processo de shielding. ${ }^{35}$ Se a ponteira do ultrassom es- 
tiver bem próxima ao eletrodo de $\mathrm{DDB}$ ( $d$ menores que $5 \mathrm{~mm}$ ), isto poderia causar um bloqueio de parte da corrente que passa entre o eletrodo de trabalho e o eletrodo auxiliar, resultando numa densidade de corrente não uniforme na superfície eletródica (shielding $)^{35} \mathrm{e}, \mathrm{com}$ isso, interferir diretamente na medida eletroquímica.

Além disso, o processo de sonoeletroxidação do carbaril acontece em potenciais mais positivos em relação ao voltamograma na ausência do ultrassom, à medida que a distância entre a ponteira e o eletrodo diminui. Esse deslocamento no valor de potencial é devido aos efeitos de cavitação. Sabe-se que as bolhas geradas por esse processo crescem próximas à superfície eletródica, tornando-se um obstáculo para o crescimento da camada de difusão e, consequentemente, dificulta a ocorrência do processo eletroquímico. ${ }^{36,37}$

Em adição, esses deslocamentos de potenciais podem também ser justificados pelo aumento da queda ôhmica. Esse fenômeno consiste numa força motriz, na forma de potencial, necessária para vencer a resistência da solução. Essa força segue a lei de Ohm e é igual ao produto da corrente em ampéres pela resistência da célula em ohms. ${ }^{35}$ Diante dos elevados valores de corrente obtidos em menores valores de $d$ (resultados da Figura 1), há um aumento da queda ôhmica e, consequentemente, o processo eletroquímico ocorre em maiores valores de potencial. É importante citar que esses deslocamentos de potenciais em medidas sonovoltamétricas também foram observados nos estudos do metilparation e do 4-nitrofenol. ${ }^{6,7}$

Em estudos da variação da $A$ e do $t$ a um valor de $d$ constante (5 $\mathrm{mm}$ ) para a oxidação de carbaril, não foi observado um aumento na intensidade de corrente dos sonovoltamogramas quando comparados ao voltamograma obtido em condições de $A=20 \%$ e $t=0 \mathrm{~s}$. Adicionalmente, neste estudo de variação de $t$, também não foi notada a degradação sonoquímica do pesticida. Com isso, as condições otimizadas para a aplicação do ultrassom na determinação voltamétrica de carbaril foram um valor de $d$ de $5 \mathrm{~mm}, A$ de $20 \%$ com aplicação do ultrassom somente durante as medidas voltamétricas.

Como consequência do uso do ultrassom na oxidação de carbaril, um aumento no transporte das espécies eletroativas até a superfície eletródica foi observado, visto que as respostas sonovoltamétricas (6-10) do sistema apresentaram intensidades de corrente 1,5 vezes maiores que aquela obtida na primeira resposta realizada em condições quiescentes (1), como mostra a Figura 2.

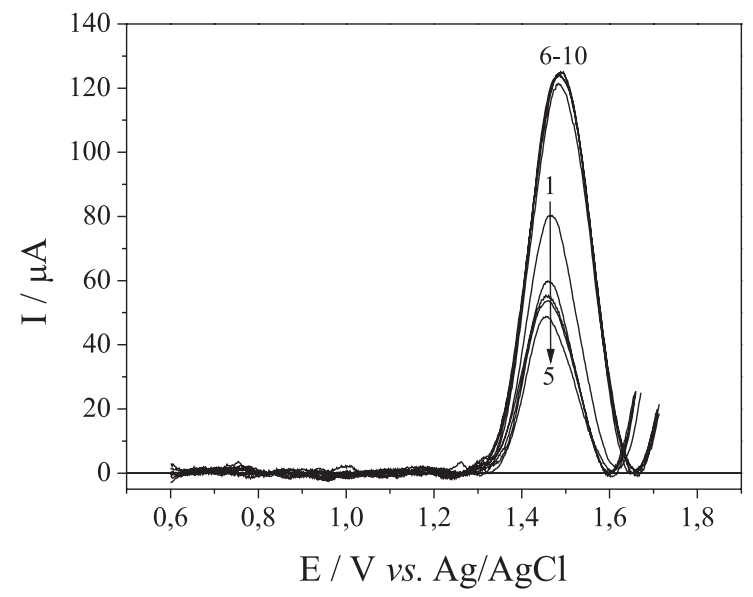

Figura 2. Voltamogramas de onda quadrada sucessivos para a oxidação de carbaril a 50,0 $\times 10^{-6} \mathrm{~mol} \mathrm{~L}^{-1}$ na ausência (1-5) e na presença do ultrassom (6-10). Condições: $\mathrm{Na}_{2} \mathrm{SO}_{4} 0,1 \mathrm{~mol} \mathrm{~L}^{-1}(\mathrm{pH}=6,0) \mathrm{comf}=300 \mathrm{~s}^{-1}, a=50 \mathrm{mV}$, $\Delta E_{s}=2 \mathrm{mV}$. Ultrassom: $d=5 \mathrm{~mm}$ e $A=20 \%$

Além disso, a Figura 2 mostra o efeito da limpeza da superfície eletródica por meio das curvas 1-5 e 6-10 obtidas sucessivamente na ausência e na presença do ultrassom, respectivamente, todas realizadas sem limpeza intermediária da superfície. ${ }^{1}$ Esse efeito ocorre por meio de colapsos cavitacionais de microbolhas produzidas pelo ultrassom. Notou-se que as medidas voltamétricas sucessivas realizadas na ausência da radiação (curvas 1-5) apresentaram uma contínua diminuição na resposta devido à adsorção e/ou insolubilização de produtos da oxidação do carbaril. Consequentemente, uma diminuição na sensibilidade do método de detecção foi observada. Por outro lado, os sucessivos sonovoltamogramas (curvas 6-10) mostraram pequenas mudanças na corrente. Esses experimentos demonstram a capacidade do ultrassom em limpar a superfície do eletrodo de diamante, permitindo o seu uso contínuo sem a necessidade de limpezas intermediárias.

Após a otimização das condições experimentais de aplicação do ultrassom, voltamogramas de onda quadrada em diferentes concentrações de carbaril foram obtidos em solução de $\mathrm{Na}_{2} \mathrm{SO}_{4} 0,1 \mathrm{~mol} \mathrm{~L}{ }^{-1}$, na ausência (Figura 3A) e na presença do ultrassom (Figura 3B). A figura inserida na Figura $3 \mathrm{~B}$ corresponde às curvas analíticas para $\mathrm{O}$ intervalo de concentrações estudado.
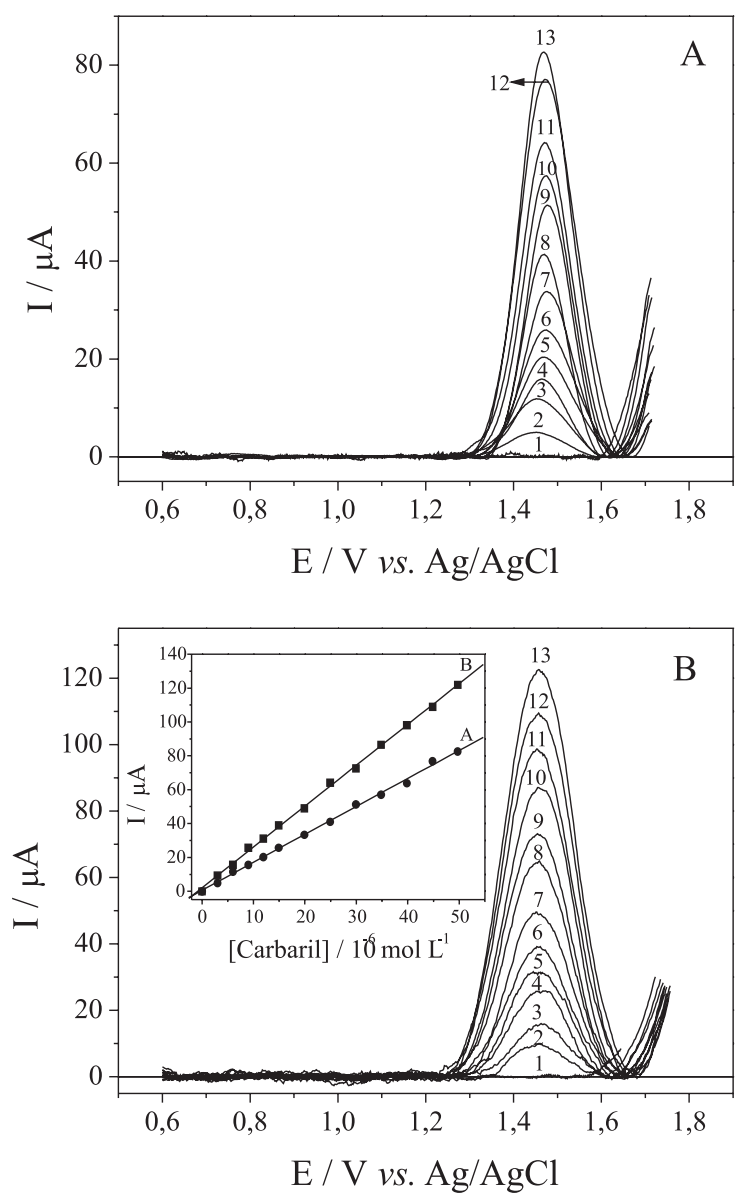

Figura 3. Voltamogramas de onda quadrada para diferentes concentrações de carbaril na ausência (A) e na presença (B) do ultrassom: 0 (1), 3,00 (2) a $50,0 \times 10^{-6} \mathrm{~mol} \mathrm{~L}^{-1}(13)$. Condições: $\mathrm{Na}_{2} \mathrm{SO}_{4} 0,1 \mathrm{~mol} \mathrm{~L}^{-1}(\mathrm{pH}=6,0) \mathrm{comf}=$ $300 \mathrm{~s}^{-1}, a=50 \mathrm{mV}, \Delta E_{\mathrm{s}}=2 \mathrm{mV}$. Ultrassom: $d=5 \mathrm{~mm}$ e $A=20 \%$. Inserção em B: curvas analíticas obtidas

Como mostrado na Tabela 1, o valor de sensibilidade obtido para a curva realizada na presença do ultrassom é 1,5 vezes maior em relação ao valor obtido em condições quiescentes. A precisão dos valores de sensibilidade, avaliada pelo cálculo do desvio padrão relativo (rsd), foi 2,0 vezes melhor para o método sonovoltamétrico 
(1,95\%) que o valor obtido pela SWV na ausência do ultrassom $(3,91 \%)$, confirmando o sinergismo produzido na combinação da irradiação de ultrassom com medidas eletroanalíticas.

Tabela 1. Parâmetros obtidos pelas curvas analíticas, valores de LD e LQ e porcentagens de recuperação para a oxidação de carbaril na ausência e na presença do ultrassom

\begin{tabular}{lcc}
\hline Parâmetros & \multicolumn{2}{c}{ Água pura } \\
\hline $\mathrm{b}\left(\mathrm{A} \mathrm{L} \mathrm{mol}{ }^{-1}\right)$ & 1,65 & sono-SWV \\
$\operatorname{rsd}^{\mathrm{A}}(\%)$ & 3,91 & 2,41 \\
$\mathrm{r}$ & 0,9990 & 1,95 \\
$\mathrm{~S}_{\mathrm{B}}(\mu \mathrm{A})$ & $8,10 \times 10^{-3}$ & 0,9995 \\
$\mathrm{LD}\left(\mu \mathrm{g} \mathrm{L}^{-1}\right)$ & 2,96 & $8,30 \times 10^{-3}$ \\
$\mathrm{LQ}\left(\mu \mathrm{g} \mathrm{L}^{-1}\right)$ & 9,87 & 2,08 \\
$\operatorname{Rec} 1^{\mathrm{B}}(\%)$ & $98 \pm 2$ & 6,92 \\
$\operatorname{Rec} 2^{\mathrm{B}}(\%)$ & $96 \pm 1$ & $98 \pm 1$ \\
\hline
\end{tabular}

${ }^{\mathrm{A}} \mathrm{n}=3$, para cada caso; ${ }^{\mathrm{B}} \mathrm{n}=3$, para cada concentração: 9,0 (Rec 1$)$ e $30,0(\operatorname{Rec} 2) \times 10^{-6} \mathrm{~mol} \mathrm{~L}^{-1}$.

A determinação do LD e do LQ foi realizada conforme o procedimento descrito na parte experimental. $\mathrm{O}$ valor do $\mathrm{LD}$ obtido na presença do ultrassom foi de $1,03 \times 10^{-8} \mathrm{~mol} \mathrm{~L}^{-1}\left(2,08 \mu \mathrm{g} \mathrm{L} \mathrm{L}^{-1}\right)$, um valor ligeiramente menor que o obtido na ausência da radiação, 1,47 $\times 10^{-8} \mathrm{~mol} \mathrm{~L}^{-1}\left(2,96 \mu \mathrm{g} \mathrm{L}^{-1}\right)$. Apesar da pequena melhora no LD, a limpeza da superfície do eletrodo de DDB providenciada pelo ultrassom foi bastante eficiente, como é claramente mostrado na Figura 2, evidenciando que a limpeza intermediária da superfície não é necessária durante a construção de curvas analíticas na presença do ultrassom. Isto diminui consideravelmente o tempo necessário na determinação analítica do pesticida, simplificando também o método de detecção. Adicionalmente, foi observado que os valores de LD e de LQ são bastante inferiores ao valor de LMR permitido para este pesticida como, por exemplo, na cultura de abacaxi (LMR $=500 \mu \mathrm{g}$ $\mathrm{kg}^{-1}$ ). Os parâmetros das regressões lineares e os valores de LD e LQ estão sumarizados na Tabela 1.

Em adição, o valor do LD determinado para o método sonovoltamétrico é menor que aquele obtido, por exemplo, por Pedrosa et al. ${ }^{19}$ que utilizaram um biossensor amperométrico baseado na AChE $\left(9,0 \mu \mathrm{g} \mathrm{L}^{-1}\right)$ e também menor que o valor obtido por Cai e $\mathrm{Du},{ }^{20}$ que construíram um sensor baseado na imobilização da AChE sobre eletrodos impressos modificados com nanotubos de carbono de paredes múltiplas $\left(4,0 \mu \mathrm{g} \mathrm{L}^{-1}\right)$.

Estudos de recuperação de carbaril em dois níveis de concentração $\left(9,0\right.$ e $\left.30,0 \times 10^{-6} \mathrm{~mol} \mathrm{~L}^{-1}\right)$ foram realizados para se avaliar a exatidão das metodologias propostas e os bons resultados obtidos estão incluídos na Tabela 1. Com isso, as metodologias desenvolvidas foram aplicadas para a detecção de carbaril numa amostra de suco de abacaxi.

\section{Determinação sonovoltamétrica de carbaril numa amostra de suco de abacaxi}

Inicialmente, medidas voltamétricas utilizando a SWV foram realizadas em soluções contendo o eletrólito suporte e alíquotas da fase orgânica provenientes da ELL (sem dopagem com carbaril) e demonstraram ausência de resíduos do pesticida na amostra estudada. Em seguida, avaliou-se a exatidão da metodologia voltamétrica, na ausência e na presença do ultrassom, por meio de estudos de recuperação do analito intencionalmente adicionado à amostra de suco de abacaxi, em níveis de concentração de 9,0 e $30,0 \times 10^{-6} \mathrm{~mol} \mathrm{~L}^{-1}$ após a etapa de ELL.

$\mathrm{Na}$ Figura 4, estão apresentados os voltamogramas e as curvas de recuperação para a concentração de $9,0 \times 10^{-6} \mathrm{~mol} \mathrm{~L}^{-1}$ de carbaril. Estudos na concentração de $30,0 \times 10^{-6} \mathrm{~mol} \mathrm{~L}^{-1}$ apresentaram perfis voltamétricos e curvas de recuperação semelhantes à concentração anterior.
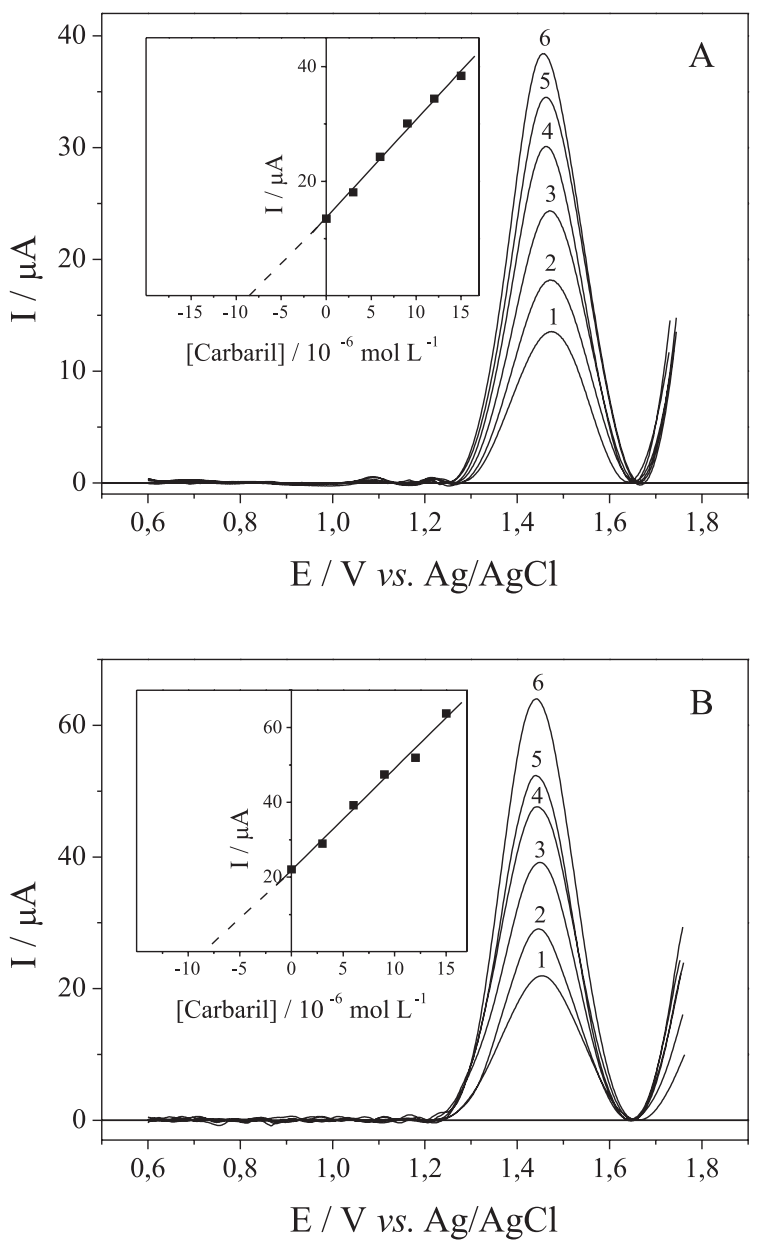

Figura 4. Voltamogramas de onda quadrada obtidos para $9,00 \times 10^{-6} \mathrm{~mol} \mathrm{~L}^{-1}$ de carbaril após ELL com acetonitrila em uma amostra de suco de abacaxi (1) e para soluções resultantes após adição de alíquotas conhecidas do composto (2-6) na ausência (A) e na presença (B) do ultrassom. Condições: $\mathrm{Na}_{2} \mathrm{SO}_{4} \mathrm{O}, 1$ $m o l L^{-1}(p H=6,0) \operatorname{com} f=300 s^{-1}, a=50 \mathrm{mV}, \Delta E_{s}=2 \mathrm{mV}$. Ultrassom: $d=5$ mm e $A=20 \%$. Inserções em B: curvas de recuperação obtidas

As adequadas porcentagens de recuperação obtidas (na faixa entre 89 e 92\%) estão listadas na Tabela 2. Esse conjunto de dados demonstra que a aplicação da SWV e da sono-SWV sobre o eletrodo de DDB são poderosas ferramentas para a determinação de carbaril em amostras de alimentos, tais como frutas.

É importante ressaltar que as curvas obtidas na ausência do ultrassom apresentadas nas Figuras 3A e 4A foram construídas com a limpeza intermediária da superfície do eletrodo de DDB e que as medidas sonovoltamétricas foram realizadas sem a limpeza intermediária da superfície. Isso destaca a importância da metodologia sonoeletroquímica desenvolvida, visto que foram obtidos resultados analíticos satisfatórios. Além disso, a metodologia desenvolvida neste trabalho se apresentou bem mais simples e rápida em relação a algumas metodologias cromatográficas desenvolvidas para a detecção analítica de carbaril. ${ }^{13,15,17}$ Nestes estudos, os autores utilizaram 
Tabela 2. Porcentagens de recuperação de carbaril, na ausência e na presença do ultrassom, em uma amostra de suco de abacaxi após procedimentos de ELL

\begin{tabular}{lcc}
\hline \multirow{2}{*}{ Recuperações } & \multicolumn{2}{c}{ Suco de Abacaxi } \\
\hline $\operatorname{Rec~1*(\% )}$ & SWV & sono-SWV \\
\hline $\operatorname{Rec~2*(\% )}$ & $89 \pm 3$ & $89 \pm 2$ \\
\hline
\end{tabular}

*n $=3$, para cada concentração: 9,0 (Rec 1) e 30,0 (Rec 2) $\times 10^{-6} \mathrm{~mol} \mathrm{~L}^{-1}$.

a cromatografia líquida de alta eficiência para a determinação do pesticida, acompanhada por complexos procedimentos de extração do analito de amostras de alimentos.

\section{CONCLUSÕES}

Os resultados evidenciaram que o ultrassom associado à SWV empregando o eletrodo de DDB é uma interessante ferramenta para a determinação do pesticida carbaril, assim como para outros pesticidas empregados na agricultura. ${ }^{6}$ Isto é devido ao aumento do transporte do material eletroativo até a superfície do eletrodo e à eficiente limpeza desta superfície, ambos os efeitos proporcionados pelo ultrassom, resultando em análises mais sensíveis, rápidas e reprodutíveis.

As metodologias na ausência e na presença do ultrassom em conjunto com um prévio procedimento de ELL foram aplicadas com sucesso para a determinação de carbaril em uma amostra de suco de abacaxi, obtendo porcentagens de recuperação do analito entre $89 \mathrm{e}$ $92 \%$. Esses métodos podem ser utilizados para a análise de resíduos de pesticidas da classe dos carbamatos em amostras de frutas, com vantagem para o método sonovoltamétrico, visto que não é necessária a limpeza intermediária da superfície eletródica.

\section{AGRADECIMENTOS}

Ao CNPq (Proc. 142930/2005-9 e 304018/2009-0) e à Fapesp (Proc. 06/50692-2) pelas bolsas e apoio financeiro concedido.

\section{REFERÊNCIAS}

1. Garbellini, G. S.; Salazar-Banda, G. R.; Avaca, L. A.; Quim. Nova 2008 , $31,123$.

2. Compton, R. G.; Eklund, J. C.; Marken, F.; Electroanalysis 1997, 9, 509.

3. Banks, C. E.; Compton, R. G.; Chem. Phys. Chem. 2003, 4, 169.

4. Compton, R. G.; Eklund, J. C.; Page, S. D.; Mason, T. J.; Walton, D. J.; J. Appl. Electrochem. 1996, 26, 775.

5. Oliveira, R. T. S.; Garbellini, G. S.; Salazar-Banda, G. R.; Avaca, L. A.; Anal. Lett. 2007, 40, 2673.

6. Garbellini, G. S.; Salazar-Banda, G. R.; Avaca, L. A.; Food Chem. 2009, 116,1029

7. Garbellini, G. S.; Salazar-Banda, G. R.; Avaca, L. A.; J. Braz. Chem. Soc. 2007, 18, 1095.

8. Codognoto, L.; Tanimoto S. T.; Pedrosa, V. A.; Suffredini, H. B.; Machado, S. A. S.; Avaca, L. A.; Electroanal. 2006, 18, 253.
9. Garbellini, G. S.; Uliana, C. V.; Pesticidas: Revista de Ecotoxicologia e Meio Ambiente 2007, 17, 29.

10. Mwanza, J.; Finley, D.; Spivey, C. L.; Graff, J. E.; Herr, D. W.; Neurotoxicology 2008, 29, 87.

11. Moreno, A. J. M.; Serafim, T. L.; Oliveira, P. J.; Madeira, V. M. C.; Chemosphere 2007, 66, 404.

12. http://portal.anvisa.gov.br/wps/wcm/connect/93b39980405ac42ab5f7f7 330f10004b/C03++Carbaril.pdf, acessada em Outubro 2010.

13. Santalad, A.; Srijaranai, S.; Burakham, R.; Glennon, J. D.; Deming, R. L.; Anal. Bioanal. Chem. 2009, 394, 1307.

14. Lal, A.; Tan, G.; Chai, M.; Anal. Sci. 2008, 24, 231.

15. Pérez-Ruiz, T.; Martínez-Lozano, C.; García, M. D.; J. Chromatogr., A 2007, 1164, 174.

16. Ito, I.; Goto, T.; Yamada, S.; Matsumoto, H.; Oka, H.; Takahashi, N.; Nakazawa, H.; Nagase, H.; Ito, Y.; J. Chromatogr., A 2006, 1108, 20.

17. Orejuela, E.; Silva, M.; J. Chromatogr., A 2003, 1007, 197.

18. March, C.; Manclus, J. J.; Jimenez, Y.; Arnau, A.; Montoya, A.; Talanta 2009, 78, 827

19. Pedrosa, V. A.; Caetano, J.; Machado, S. A. S.; Bertotti, M.; Sensors 2008, 8,4600 .

20. Cai, J.; Du, D. A.; J. Appl. Electrochem. 2008, 38, 1217.

21. De Albuquerque, Y. D. T.; Ferreira, L. F.; Anal. Chim. Acta 2007, 596, 210.

22. Del Carlo, M.; Mascini, M.; Pepe, A.; Diletti, G.; Compagnone, D.; Food Chem. 2004, 84, 651.

23. Rao, T. N.; Loo, B. H.; Sarada, B. V.; Terashima, C.; Fujishima, A.; Anal. Chem. 2002, 74, 1578.

24. Mccreery, R. L.; Chem. Rev. 2008, 108, 2646.

25. Salazar-Banda, G. R.; Andrade, L. S.; Nascente, P. A. P.; Pizani, P. S.; Rocha-Filho, R. C.; Avaca, L. A.; Electrochim. Acta 2006, 51, 4612.

26. Suffredini, H. B.; Machado, S. A. S.; Avaca, L. A.; J. Braz. Chem. Soc. 2004, 15, 16.

27. Suffredini, H. B.; Pedrosa, V. A.; Codognoto, L.; Machado, S. A. S.; Rocha-Filho, R. C.; Avaca, L. A.; Electrochim. Acta 2004, 49, 4021.

28. Compton, R. G.; Foord, J. S.; Marken, F.; Electroanalysis 2003, 15, 1349.

29. Goeting, C. H.; Foord, J. S.; Marken, F.; Compton, R. G.; Diamond Relat. Mater. 1999, 8, 824.

30. Compton, R. G.; Marken, F.; Goeting, C. H.; Mckeown, R. A. J.; Foord, J. S.; Scarsbrook, G.; Sussmann, R. S.; Whitehead, A. J.; Chem. Commun. 1998, 18, 1961.

31. Caetano, J.; Machado, S. A. S.; Sens. Actuators, B 2008, 129, 40.

32. Salazar-Banda, G. R.; Andrade, L. S.; de Carvalho, A. E.; FatibelloFilho, O.; Rocha-Filho, R. C.; Avaca, L. A.; Anal. Chem., submetido.

33. Mocak, J.; Bond, A. M.; Mitchell, S.; Scollary, G.; Pure Appl. Chem. 1997, 69, 297

34. Marken, F.; Compton, R. G.; Ultrason. Sonochem. 1996, 3, S131.

35. Bard, A. J.; Faulkner, L. R.; Electrochemical methods: fundamentals and applications, $2^{\text {nd }}$ ed., Wiley: New York, 2001.

36. Maisonhaute, E.; White, P. C.; Compton, R. G.; J. Phys. Chem. B 2001, 105, 12087.

37. Maisonhaute, E.; Brookes, B. A.; Compton, R. G.; J. Phys. Chem. B 2002, 106, 3166. 